\title{
Continued in vitro cefazolin susceptibility in methicillin-susceptible Staphylococcus aureus
}

Benjamin H. Gern' ${ }^{1}$, Alexander L. Greninger², Scott J. Weissman' ${ }^{1}$, Jennifer R. Stapp ${ }^{3}$, Yue Tao ${ }^{4}$ and Xuan Qin ${ }^{1,2,3^{*}}$ (1)

\begin{abstract}
Objectives: In vitro trends of cefazolin and ceftriaxone susceptibilities from pediatric clinical isolates of methicillinsusceptible Staphylococcus aureus (MSSA) between 2011 and 2016 were analyzed for surveillance.

Methods: Our laboratory continues to use agar disk diffusion for staphylococcal susceptibilities applying Clinical Laboratory Standard Institute's 2012 breakpoints.

Results: A total of 3992 MSSA clinical isolates in the last 6 years were analyzed for their in vitro cefazolin and ceftriaxone susceptibilities. While all MSSA isolates exhibited cefazolin susceptibilities within the "susceptible" zone range, there have been a proportion of isolates with ceftriaxone susceptibilities falling in "intermediate" zones, ranging from $2.6 \%$ in 2011 to $8.3 \%$ in 2016.

Conclusions: Cefazolin continues to be the recommended agent for MSSA treatment at our institution, reflected by the finding that only $2 \%$ (6/321) of patients who received ceftriaxone as definitive therapy for MSSA bacteremia during the study period. We have confirmed the cefoxitin-predicted MSSA susceptibility to cefazolin, but have found concerning drifts in ceftriaxone susceptibilities by continued in vitro monitoring over the last 6 years.
\end{abstract}

Keywords: Staphylococcus aureus, MSSA, Cefazolin, Ceftriaxone, MIC

\section{Background}

The development of resistance of Staphylococcus aureus to $\beta$-lactam antibiotics has been well-characterized starting from the first uses of penicillin [1]. This first occurred starting with production of penicillinase, then $m e c A$ - and vanA-determined mechanisms to alter the components of cell wall synthesis $[2,3]$. The gene $m e c A$ confers the majority of resistance to penicillinase-stable $\beta$-lactams [4]. For over 20 years, the Clinical Laboratory Standard Institute (CLSI) has included all $\beta$-lactam agents, including all classes of cephalosporins against staphylococcal species, with minimum inhibitory concentration (MIC) or zone size breakpoint recommendations [5]. In January

\footnotetext{
*Correspondence: xuan.qin@seattlechildrens.org

1 Department of Pediatrics, Seattle Children's Hospital, University of Washington, Seattle, WA, USA

Full list of author information is available at the end of the article
}

2013, CLSI eliminated all $\beta$-lactam antibiotic breakpoints for methicillin-susceptible $S$. aureus (MSSA), except oxacillin, cefoxitin, penicillin, and ceftaroline [6]. This recommendation derives from the understanding that susceptibility to antistaphylococcal $\beta$-lactams can be inferred using the above agents. Following this recommendation, there have been a few small in vitro and clinical studies that have examined the question of "inferred susceptibility" for MSSA [7-10]. This study intends to use our existing in vitro susceptibility data to inform future practices.

\section{Methods}

The agar disk diffusion method has been consistently used in our laboratory for susceptibility testing of all Staphylococcus spp. for more than 20 years. The susceptibility panel included antibiotic disks (Remel, USA): penicillin (10 units), oxacillin (1 $\mu \mathrm{g}$, prior to 2011$)$ or cefoxitin 
(30 $\mu \mathrm{g}$ after 2011), amoxicillin/clavulanate $(20 / 10 \mu \mathrm{g})$, cefazolin $(10 \mu \mathrm{g})$, ceftriaxone $(10 \mu \mathrm{g})$, meropenem $(10 \mu \mathrm{g})$, gentamicin $(10 \mu \mathrm{g})$, erythromycin $(15 \mu \mathrm{g})$, clindamycin $(2 \mu \mathrm{g})$, ciprofloxacin $(5 \mu \mathrm{g})$, rifampin $(5 \mu \mathrm{g})$, sulfamethoxazole-trimethoprim (SMX-TMP, 1.25/23.75 $\mu \mathrm{g}$ ), linezolid $(30 \mu \mathrm{g})$, and vancomycin $(30 \mu \mathrm{g}$ disk prior to 2010 then MIC by Etest [BioMerieux, France]). Nitrofurantoin $(300 \mu \mathrm{g})$ is tested and reported in urine isolates only, while erythromycin and clindamycin are not reported in urine isolates. Our lab has continued to test for, and report, cefazolin and ceftriaxone susceptibility for MSSA isolates using the 2012 CLSI breakpoints [5]. According to CLSI recommendations, we measure the zone diameters (except for trimethoprim-sulfamethoxazole) by holding the Petri plate a few inches above a black background illuminated with reflected light, except for linezolid, which was read with transmitted light [11]. Weekly quality controls are performed using S. aureus ATCC 25923 for disk diffusion and S. aureus ATCC 29213 for Etest tested on Mueller-Hinton agar (Remel, USA) with acceptable in-range limits (Additional file 1: Figure S1). For this study, repeat isolates on the same patients within a calendar year were excluded, regardless of specimen source.

We retrospectively examined the cefazolin and ceftriaxone susceptibility profiles of all MSSA isolates in our lab between 2011 and 2016. To assess ceftriaxone use among patients with MSSA infections, we used records maintained by our Antimicrobial Stewardship Program (ASP) to identify a subset of patients that had MSSApositive blood cultures and received ceftriaxone during this period. We then reviewed patient medical records to determine whether ceftriaxone was used as definitive therapy.

\section{Results and discussion General findings}

A total of 3992 MSSA isolates tested between 2011 and 2016 were included in the susceptibility analysis. Using 2012 CLSI criteria, we confirmed that cefazolin remained an active agent, with all zone interpretive range confined above the "susceptible range of $\geq 19 \mathrm{~mm}$ (susceptible: $\geq 18 \mathrm{~mm}$ ), and none in "intermediate" range of 15-17 mm (data not shown). We also found that a proportion of MSSA isolates produced ceftriaxone susceptibilities in "intermediate" zones ranges (14-20 mm) over the 6 year period, from $2.6 \%$ in 2011 to $8.3 \%$ in 2016 with the highest of $15.5 \%$ in 2014 (Fig. 1). None of the MSSA isolates produced ceftriaxone zone measurements in the "resistant" range (Fig. 1).

When ceftriaxone susceptibilities were further broken down by site of culture, we found that while blood, respiratory, and wound/deep tissue infections had similar proportion of MSSA isolates with ceftriaxone "intermediate" at $6.30,6.82$, and $6.15 \%$ respectively, the MSSA strains isolated from urine cultures showed higher rate of non-susceptibility to ceftriaxone (11.27\%, Table 1$)$. From the same period, methicillin-sensitive coagulase-negative Staphylococcus species (MS-CoNS, $\mathrm{n}=673$ ), were observed to be $8.0 \%$ in "intermediate" range to ceftriaxone. This fraction reduced to $3.1 \%$ when S. saprophyticus isolates $(\mathrm{n}=80)$ were excluded, which accounted for the majority of MS-CoNS ceftriaxone non-susceptibility in our study. No "intermediate" range susceptibility to cefazolin was observed in MS-CoNS isolates, which was $100 \%$ sensitive over the course of our study (data not shown).

\section{In vitro susceptibility patterns}

Upon closer examination, growth patterns of MSSA isolates around ceftriaxone disks characteristically meeting the CLSI description of a "beach" type of heterogeneous inhibitory zone, as opposed to a "cliff" inhibitory zone around cefazolin (Fig. 2) [11]. Post hoc crosscheck of zone characteristics around of cefazolin and ceftriaxone disks on clinical-convenient samples $(n=153)$ have confirmed the distinct inhibitory zone characteristics between the two as shown in Additional file 1: Figure S2.

\section{Clinical practices and current state of in vitro testing}

During the study period of 2011-2016, 321 patients with MSSA bacteremia were identified, in which only 6 patients received ceftriaxone as definitive therapy (after final susceptibility reports) without evidence of treatment failure.

Our institutional Antimicrobial Stewardship Committee has implemented a Microbiology Result Comment of " $\beta$-lactams like cefazolin and nafcillin are superior to vancomycin for treatment of MSSA" since mid-2013. This has, in part, resulted in our low number of cases where ceftriaxone was used as the definitive therapy. This hinders our ability to assess the clinical implications of ceftriaxone non-susceptibility (or "intermediate"), as all patients who received ceftriaxone as definitive therapy had known-susceptible isolates due to our cephalosporin susceptibility testing and reporting practices.

Ceftriaxone is not a first-line agent for MSSA infections, but its favorable dosing parameters often makes it a favorable choice in outpatient management. While there are no randomized controlled trials examining its effectiveness in treating MSSA infections, there have been small observational studies examining clinical outcomes, though it is not apparent that these studies tested for in vitro ceftriaxone non-susceptibility. One retrospective study from Israel investigated treatment of MSSA bacteremia in 541 patients with different $\beta$-lactams, using 


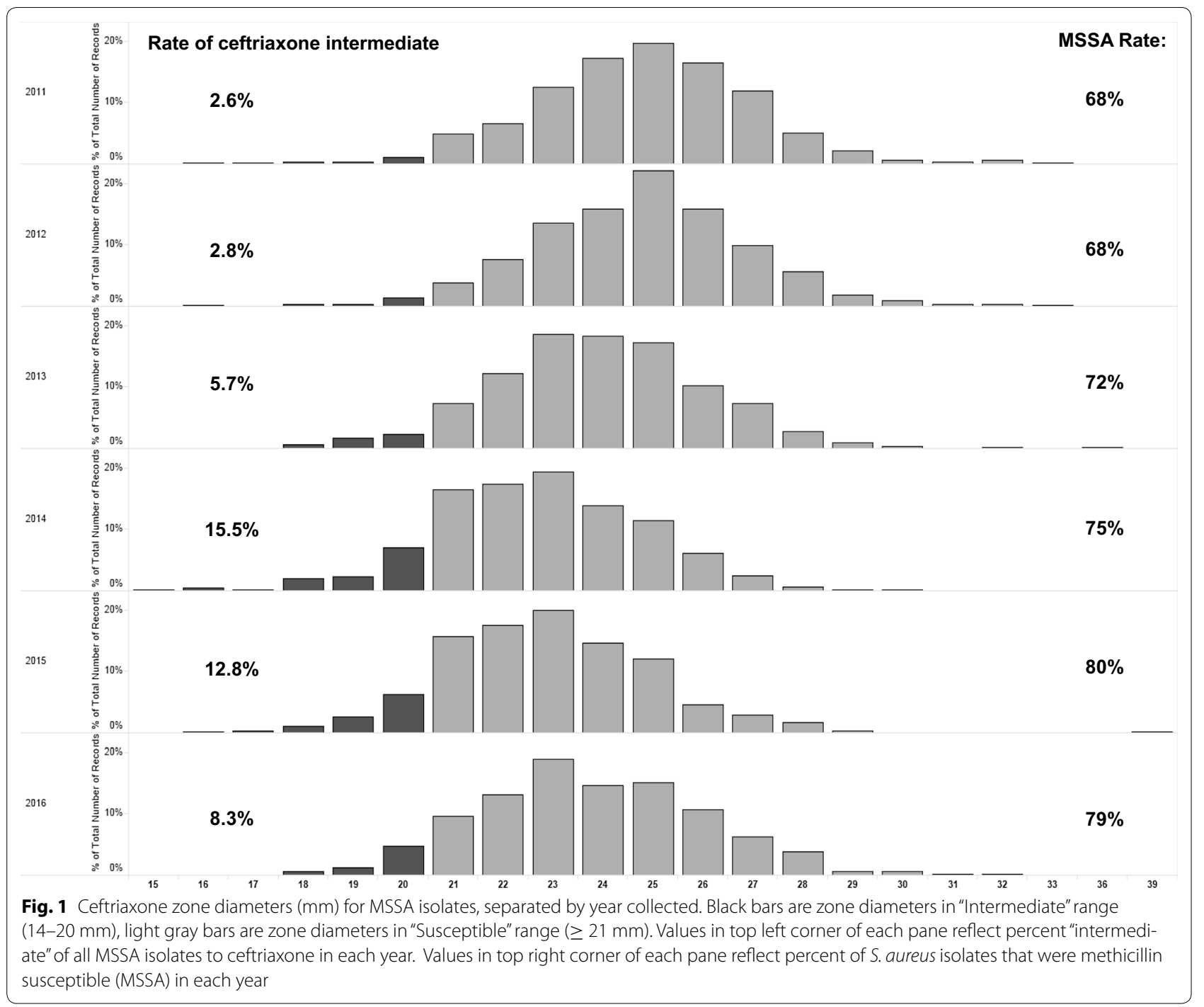

Table 1 Ratio of ceftriaxone susceptibilities against the 6-year MSSA isolates based on specimen source

\begin{tabular}{lcc}
\hline Specimen type (group) & \multicolumn{2}{c}{ Ceftriaxone susceptibility } \\
\cline { 2 - 3 } & Intermediate (\%) & Susceptible (\%) \\
\hline Blood $(n=349)$ & 6.30 & 93.70 \\
Cerebrospinal fluid $(n=22)$ & & 100.00 \\
Osteoarticular $(n=33)$ & 3.03 & 96.97 \\
Respiratory tract $(n=1642)$ & 6.82 & 93.18 \\
Urine $(n=213)$ & 11.27 & 88.73 \\
Wound and deep tissue infections & 6.15 & 93.85 \\
$(n=3348)$ & & \\
\hline
\end{tabular}

"inferred susceptibility" [9]. Ceftriaxone therapy was associated with higher 30-day adjusted mortality odds, though this did not hold with 90-day mortality. Another retrospective cohort study from Texas investigated ceftriaxone versus cefazolin therapy for invasive MSSA infections in 122 patients who received outpatient parenteral antibiotic therapy, finding similar clinical outcomes and adverse events [8]. Lastly, investigators from Missouri retrospectively compared ceftriaxone versus oxacillin in 124 patients with MSSA osteomyelitis and/or septic arthritis, finding similar clinical outcomes, though acknowledging fewer medication side effects with ceftriaxone treatment [10]. These studies highlight the need for further clinical trials.

The low historical prevalence of MSSA resistance to cefazolin, ceftriaxone and other antistaphylococcal $\beta$-lactams is well-described [12], and reflected in the recommendation to remove most $\beta$-lactam breakpoints from the 2013 CLSI (M100-S23) guidelines. As a result of this 


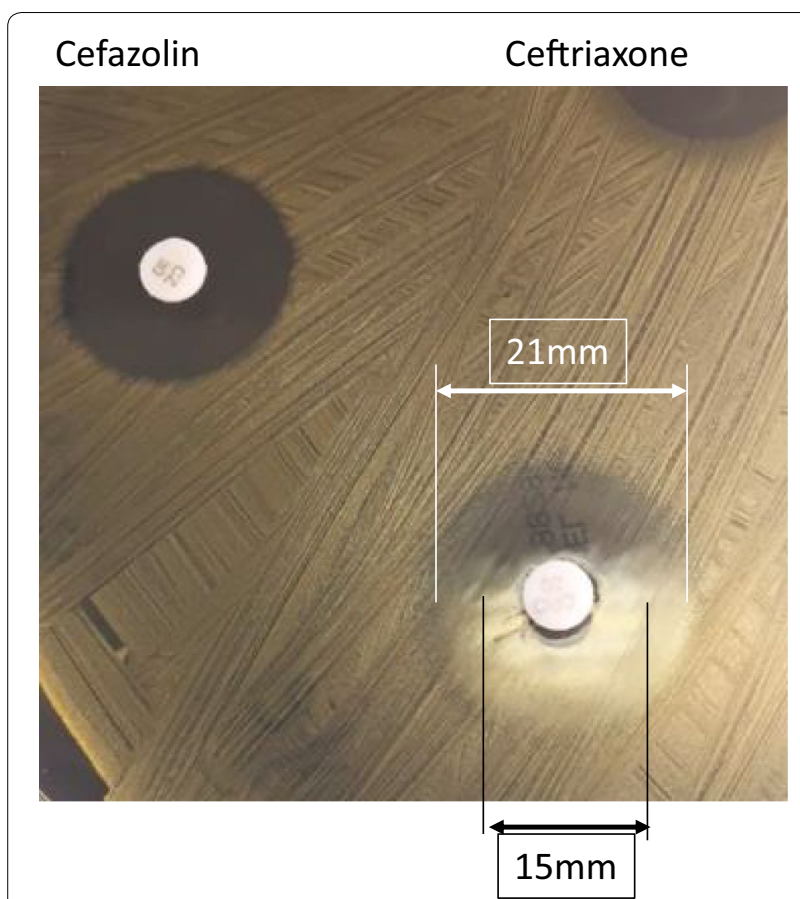

Fig. 2 Characteristic "Cliff" versus "Beach" inhibitory zones associated with cefazolin and ceftriaxone respectively by disk diffusion method against MSSA isolates

recommendation, cephalosporin agents such as cefazolin and ceftriaxone have been eliminated from most commercial antistaphylococcal susceptibility panels for surveillance information. Our data from continued testing of cefazolin and ceftriaxone using agar disk diffusion alone, without a precise MIC correlation clearly has limitations. It is possible there is a $m e c A$-independent mechanism in MSSA and MS-CoNS conferring their reduced susceptibility to certain $\beta$-lactams, similar to the ever-emerging multitude of resistance in Gram-negative bacteria. This may need to be addressed for surveillance purposes. Interestingly, ceftriaxone non-susceptibility was not as pronounced in MS-CoNS, except for S. saprophyticus, which has a low overall prevalence of $m e c A$ positivity [13].

\section{Conclusions}

This study affirms that cefazolin should continue to be the first line choice for the treatment of MSSA infections, and its inferred in vitro susceptibility from cefoxitin is still accurate. Our observed drift in ceftriaxone in vitro susceptibilities serves as an awareness call for closer surveillance. Additional studies at other institutions are necessary to determine if this trend is wide-spread. Consistent with CLSI recommendations, laboratory testing of antimicrobial susceptibility not only plays a role in patient care but also epidemiology surveillance for emergence of resistance.

\section{Additional file}

Additional file 1: Figure S1. Quality control performances of weekly cefoxitin, cefazolin, and ceftriaxone disk diffusion the study period. Figure S2. Post hoc measurement of zones of inhibition generated from ceftriaxone and cefazolin disks against clinical isolates $(n=153)$ of MSSA. Heterogeneous or "beach"-type of zone phenotypes around ceftriaxone disk could be sized typically by either at $\sim 80 \%$ growth inhibition or at the complete growth inhibition (Note: The measurement at the complete growth inhibition has been the standard for susceptibility interpretations). Insert graph shows "cliff"-type of homogeneous zone measurements around cefazolin disk.

\section{Abbreviations}

MSSA: methicillin-susceptible Staphylococcus aureus; MIC: minimum inhibitory concentration; MS-CoNS: methicillin-sensitive coagulase-negative Staphylococcus species; ASP: Antimicrobial Stewardship Program.

\section{Authors' contributions}

Drs. BHG, ALG, and XQ contributed to the study design and manuscript writing. Drs. SJW and YTcontributed to data analysis. Ms. JRJ contributed to data collection and organization. All authors read and approved the final manuscript

\section{Author details}

${ }^{1}$ Department of Pediatrics, Seattle Children's Hospital, University of Washington, Seattle, WA, USA. ${ }^{2}$ Seattle Children's Microbiology Laboratory, Seattle, USA. ${ }^{3}$ Department of Laboratory Medicine, University of Washington, Seattle, WA, USA. ${ }^{4}$ Present Address: Shanghai Children's Medical Center, Translational Research Institute, Shanghai Jiao Tong University School of Medicine, Shanghai 200127, China.

\section{Acknowledgements}

We thank Seattle Children's Microbiology team for their high quality performance and quality documentation of susceptibility control results. We thank Mr. Paul Hiraiwa for his thorough investigation of Laboratory Information System records for data integrity.

\section{Competing interests}

All authors approved the current version of the manuscript for submission. All authors have no any commercial affiliations, are not consultants and do not have stock or equity interests, and patent-licensing arrangements that could be considered to pose a competing of interests regarding the submitted article.

\section{Availability of data and materials}

Susceptibility data used for this study is available electronically.

\section{Ethics approval and consent to participate}

This study was approved by the Institutional Review Board, Seattle Children's Hospital.

\section{Funding}

Dr. Benjamin H. Gern received an award by Academic Pediatric Infectious Disease NIH Training Grant:T32 HD007233 during the study period.

\section{Patient consent to participate}

Not applicable.

\section{Publisher's Note}

Springer Nature remains neutral with regard to jurisdictional claims in published maps and institutional affiliations.

Received: 12 October 2017 Accepted: 12 February 2018

Published online: 20 February 2018 


\section{References}

1. Kirby WM. Extraction of a highly potent penicillin inactivator from penicillin resistant Staphylococci. Science. 1944;99:452-3.

2. Barber M. Methicillin-resistant staphylococci. J Clin Pathol. 1961;14:385-93.

3. Chang S, Sievert DM, Hageman JC, Boulton ML, Tenover FC, Downes FP, Shah S, Rudrik JT, Pupp GR, Brown WJ, Cardo D, Fridkin SK, VancomycinResistant Staphylococcus aureus Investigative T. Infection with vancomycin-resistant Staphylococcus aureus containing the vanA resistance gene. N Engl J Med. 2003;348:1342-7.

4. Chambers HF. The changing epidemiology of Staphylococcus aureus? Emerg Infect Dis. 2001;7:178-82.

5. CLSI. Performance standards for antimicrobial susceptibility testing. M100-S22. Wayne: Institute CaLS, Clinical and Laboratory Standards Institute; 2012.

6. CLSI. Performance Standards for Antimicrobial Susceptibility Testing M100-S23. Wayne: Institute CaLS, Clinical and Laboratory Standards Institute; 2013.

7. Phe K, Dao D, Palmer HR, Tam VH. In vitro ceftriaxone susceptibility in methicillin-susceptible Staphylococcus aureus. Antimicrob Agents Chemother. 2015;59:1370
8. Winans SA, Luce AM, Hasbun R. Outpatient parenteral antimicrobial therapy for the treatment of methicillin-susceptible Staphylococcus aureus: a comparison of cefazolin and ceftriaxone. Infection. 2013;41:769-74.

9. Paul M, Zemer-Wassercug N, Talker O, Lishtzinsky Y, Lev B, Samra Z, Leibovici $L$, Bishara J. Are all beta-lactams similarly effective in the treatment of methicillin-sensitive Staphylococcus aureus bacteraemia? Clin Microbiol Infect. 2011;17:1581-6.

10. Wieland BW, Marcantoni JR, Bommarito KM, Warren DK, Marschall J. A retrospective comparison of ceftriaxone versus oxacillin for osteoarticular infections due to methicillin-susceptible Staphylococcus aureus. Clin Infect Dis. 2012:54:585-90.

11. CLSI. Performance Standards for Antimicrobial Susceptibility Testing. M100. Wayne: Institute CaLS; 2017

12. Dien Bard J, Hindler JA, Gold HS, Limbago B. Rationale for eliminating Staphylococcus breakpoints for beta-lactam agents other than penicillin, oxacillin or cefoxitin, and ceftaroline. Clin Infect Dis. 2014;58:1287-96.

13. Ferreira AM, Bonesso MF, Mondelli AL, Camargo CH, Cunha Mde L. Oxacillin resistance and antimicrobial susceptibility profile of Staphylococcus saprophyticus and other Staphylococci isolated from patients with urinary tract infection. Chemotherapy. 2012;58:482-91.

\section{Submit your next manuscript to BioMed Central and we will help you at every step:}

- We accept pre-submission inquiries

- Our selector tool helps you to find the most relevant journal

- We provide round the clock customer support

- Convenient online submission

- Thorough peer review

- Inclusion in PubMed and all major indexing services

- Maximum visibility for your research

Submit your manuscript at www.biomedcentral.com/submit 